\title{
Modelo teórico a priori para caracterizar la competencia matemática comunicar en el aprendizaje de la circunferencia ${ }^{1}$
}

\author{
Priori theoretical model to characterize the mathematical \\ learning to communicate in girth
}

\section{Priori modelo teórico para caracterizar a aprendizagem matemática para comunicar-se em perímetro}

Recibido: mayo de 2013

Aceptado: agosto de 2013
Dawson Didier Cortés Joven ${ }^{2}$

Doris Loaiza Ferla ${ }^{3}$

Luis Emiro Ramírez Gómez ${ }^{4}$

\section{Resumen}

El propósito de este trabajo es mostrar aspectos relacionados entre las tareas, los procesos matemáticos y los niveles de complejidad con la actividad matemática de aprendizaje de la Circunferencia articulados en un modelo teórico a priori (MTAP) involucrando, además de los aspectos cognitivos, los afectivos y de tendencia de acción que implica una pragmática de uso social de la misma competencia. El MTAP se aplicó con estudiantes de grado noveno de la I.E. Dante Alighieri de San Vicente del Caguán, el cual permite caracterizar la competencia matemática comunicar, mediante el análisis de capacidades, descriptores y actuaciones de los participantes, enfocados desde el constructo de la comunicación, a partir de la interacción social en el aula.

Palabras clave: Modelo teórico a priori; competencia matemática comunicar; matemáticas escolares; geometría; formas geométricas; la circunferencia; otras nociones de Educación Matemática; fines sociales.

\section{Abstract}

The purpose of this paper is to show aspects between tasks, mathematical processes and levels of complexity with learning mathematical activity Circumference articulated a priori theoretical model (MTAP) involving, in addition to the cognitive, affective and action tendency that involves a pragmatic social use of the same competition. The MTAP was applied with ninth grade students EI Dante Alighieri of San Vicente, which allows to characterize communicate mathematical competence through analysis capabilities,

1 Artículo de Investigación.

2 Universidad de la Amazonia, Maestría en Ciencias de la Educación con Énfasis en Didáctica de la Matemática. Contacto: dawsondidier@gmail.com

3 Universidad de la Amazonia, Maestría en Ciencias de la Educación con Énfasis en Didáctica de la Matemática. Contacto: dorisilla2009@hotmail.com

4 Universidad de la Amazonia, Maestría en Ciencias de la Educación con Énfasis en Didáctica de la Matemática. Contacto: luisemiro2004@yahoo.es 
descriptors and actions of the participants, focused from the construct of communication from social interaction in the classroom.

Key words: Theoretical model a priori, mathematical competence communicate; school mathematics, geometry, geometric forms, the circle, other notions of Mathematics Education; social purposes.

\section{Resumo}

O objetivo deste artigo é mostrar os aspectos entre as tarefas, os processos matemáticos e níveis de complexidade com a aprendizagem Circunferência atividade matemática articulou um modelo teórico a priori (MTAP), envolvendo, além do cognitivo, afetivo e tendência de ação que envolve um uso social pragmática da mesma competição. O MTAP foi aplicado com a nona série estudantes EI Dante Alighieri de São Vicente, o que permite caracterizar comunicar competência matemática através de capacidades de análise, descritores e ações dos participantes, com foco na construção de uma comunicação da interação

Palavras chave: Modelo teórico a priori, matemática competência comunicar; matemática escolar, geometria, formas geométricas, o círculo, outras noções de Educação Matemática; fins sociais.

\section{Presentación del problema}

Algunos aspectos que se abordan en el problema del desarrollo de competencias matemáticas tiene que ver con la naturaleza individual de las mismas, que se explica desde las particularidades del sujeto que aprende matemáticas $o$, como se postula, también involucra variables de naturaleza social y cultural que inciden en los sujetos, los contextos y en la vida misma de una comunidad de aprendizaje como es la clase de matemáticas.

El dominio del conocimiento matemático bajo esta perspectiva sociocultural, como una actividad social (Jaramillo, 2011), que involucra a los sujetos en una interacción, es importante en la medida que los estudiantes desarrollen capacidades en la construcción del significado matemático, para que sean compartidos socialmente mediante procesos de comunicación y se respete así, los distintos saberes constituidos por los diversos grupos socioculturales al interior de los mismos.

Es por lo anterior, que el diseño de las tareas permite movilizar en el aula de clase los aspectos comunicativos tanto orales como escritos involucrando variables sociales y culturales en el desarrollo de los procesos matemáticos. De esta manera consideramos necesario y útil para las intenciones del trabajo por competencias, asumir esta perspectiva gracias a que postula un conocimiento construido y compartido social y culturalmente; además, socialmente útil y a manera de preocupación como ayuda para consolidar una comunidad en educación matemática bajo este tipo de perspectiva teórica.

Esta utilidad social de las matemáticas es clave que el profesor la comprenda y la asuma para promover desde sus prácticas de enseñanza una pragmática de uso, aprendizajes situados y solución de problemas contextualizados.

El MTAP de competencia matemática comunicar, recoge en el análisis de las actuaciones de los participantes el trabajo en los aspectos cognitivos, afectivos y de tendencia de acción (D’amore, Díaz \& Fandiño, 2008), mediante la articulación de las tareas y los procesos matemáticos con la actividad matemática del estudiante, valorando la calidad de su aprendizaje y así, caracterizar la movilización de la competencia, no sólo desde el aporte que hacen los niveles de complejidad asociados a la evaluación del aspecto cognitivo de la competencia, sino desde 
el trabajo de aula, en la cual el docente está llamado, a contribuir a generar procesos de interacción entre los sujetos para que contribuyan al desarrollo de aspectos afectivos, volitivos, éticos, metacognitivos y de pragmática de uso de la competencia matemática

Es necesario incorporar al currículo procesos matemáticos dotados de una estructura que permitan sustentar y caracterizar las competencias matemáticas, en un desarrollo de actividades matemáticas, mediante el empleo de modelos basados en el enfoque competencial. El MTAP es una buena estrategia didáctica que asume descriptores para los diversos aspectos y procesos matemáticos presentes en la CMC, permitiendo la valoración y la caracterización de los mismos mediante la actividad de aprendizaje de los estudiantes, evidenciando avances en el desarrollo de su competencia mediante el paso de un nivel creciente de complejidad a otro.

Por todo lo anterior, se propone una pregunta con la cual se quiere contribuir a la reflexión y permitir de alguna manera dar respuesta a planteamientos que se derivan de lo descrito anteriormente ¿Cómo caracterizar la competencia matemática comunicar asociada al aprendizaje del objeto matemático circunferencia, en estudiantes de educación básica secundaria?

\section{Referentes conceptuales}

Conceptualizar la competencia matemática comunicar (CMC), no es fácil, ni aparece como tal, en estudios que hasta el momento se tenga de antecedentes, pero si se puede incluir como referencia para su conceptualización, diversos fenómenos que aparecen en el estudio de la interacción en el aula de matemáticas, donde se involucren procesos comunicacionales y elementos que componen de manera general la competencia; tales como, los planteados por OCDE-PISA (2006), Bishop (2005), Rico y Lupiañez (2008), Asfard (2008), D’amore, Díaz y Fandiño (2008), Rojas \& Solar (2011), y Arévalo (2012). Los anteriores estudios sirvieron para asumir en la investigación la CMC de la siguiente manera:
La CMC desarrolla en el estudiante el proceso de la negociación para animar a los estudiantes a participar mayormente en un discurso matemático que genere el desarrollo de aspectos primordiales de la comunicación escrita y verbal, convirtiéndose en un miembro activo y participante de la comunidad de aprendizaje, compartiendo y desarrollando el significado matemático en situaciones contextuales, y utilizarlo socialmente desde la relación entre factores meta-cognitivos, afectivos y volitivos.

Hacen parte de la composición del MTA de la CMC, los aspectos mencionados como componentes de la competencia matemática, referidos por (D’amore, Díaz y Fandiño, 2008) : cognitivo (relacionado con el estudio del objeto matemático, Circunferencia), el afectivo (involucrado al proceso matemático de la motivación) y de tendencia a la acción (analizado con el proceso matemático de la dedicación), combinado con los expuestos por Solar (2009): Procesos matemáticos, Tareas y Niveles de Complejidad. Para el caso del aspecto cognitivo se involucran los procesos matemáticos, de Participación, Negociación y Actividad discursiva, como procesos propios de la interacción social en el aula de matemáticas como entes de la actividad comunicacional que permite construir significados matemáticos, comunicarlos, compartirlos y desarrollarlos como estudiantes participantes miembros activos de una comunidad de aprendizaje (Bishop, 2005 y Asfard, 2008), y que de alguna manera, permite transversalizar lo cognitivo con los procesos metacognitivos, afectivos $y$ emocionales puestos en marcha, con las actuaciones de los participantes. Tal como se puede ver en la siguiente figura.

Figura 1. Aspectos del proceso comunicativo presente en la interacción social en el aula para compartir y desarrollar el significado matemático

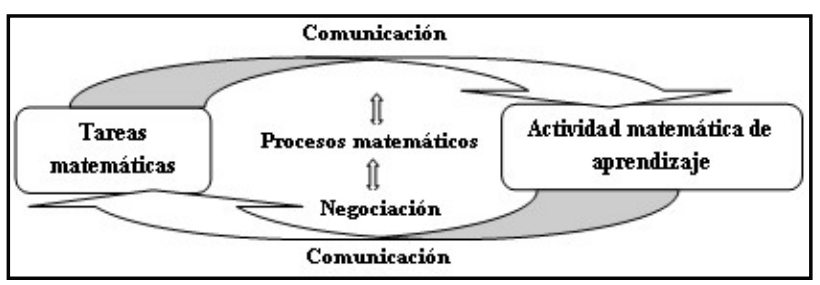

Fuente: Elaboración propia 
El objeto matemático que va a dinamizar esta relación es la Circunferencia. Para su tratamiento se tuvo en cuenta a Gómez (2002) en lo correspondiente al componente análisis de contenido que expone el autor cuando trata el análisis didáctico en su estudio "Análisis didáctico y diseño curricular en matemática. En él se hace un recorrido por la estructura conceptual, sistemas de representación y su fenomenología desde lo cotidiano y lo matemático. Para su estudio y en el diseño de las tareas se tienen en cuenta aspectos relacionados con la conceptualización, los elementos y algunas de sus propiedades.

\section{Metodología}

La metodología de investigación en la que se inscriben el análisis de los resultados es de carácter cualitativo, orientada en la postura sociocultural (Jaramillo, 2011) de naturaleza aplicada. Los estudiantes focalizados para la investigación fueron 25 estudiantes del grado 901 de la I.E.Dante Alighieri. y el método de investigación escogido para permitir el análisis fue el estudio de caso, para el cual, se tuvieron en cuenta los episodios de clase más significativos, correspondiente al trabajo realizado por cuatro estudiantes seleccionados, que fueron tenidos en cuenta para valorar las capacidades, descriptores y actuaciones, durante la actividad matemática y describir así la caracterización de la CMC. La información se recogió de tres clases y se apoyó de algunas técnicas de investigación, que sirvieron para afianzar la recolección y posterior análisis de la información, tales como videos, audios, pruebas pilotos, entrevistas, cuestionarios y análisis de documentos y materiales que fueron utilizados en el muestreo para su respectiva codificación. El profesor del área actúo al mismo tiempo como investigador.

\section{Análisis de datos}

Para el análisis de los datos se tuvo en cuenta: a) la descripción de la dinámica en el aula, en la que se cuenta el desarrollo de la actividad matemática para cada una de las clases grabadas, mostrando el objetivo específico de la tarea y el nivel creciente de complejidad de cada situación matemática, b) las actuaciones de los participantes, identificadas en el proceso del análisis de las situaciones matemáticas desarrolladas en las actividades de aula, en esta parte se caracterizo la CMC, a partir de los descriptores para cada proceso matemático del MTAP, teniendo en cuenta los episodios de clase seleccionados donde intervinieron los cuatro estudiantes objeto de estudio, y c) un análisis general de dicha interacción ${ }^{5}$, que concluye con los aspectos que permitieron la caracterización de la CMC.

\section{Conclusiones}

El estudiante realizó actividad matemática resolviendo tareas, desarrollando procesos matemáticos que le permitieron comunicar, el proceso y el producto de su actividad, la valoración de la calidad de estos procesos, mediante sus actuaciones articuladas con unos descriptores propios del proceso de la comunicación, actuaciones individualizadas que permitieron validar el constructo del significado matemático dentro del grupo. El estudiante-participante, logro movilizar procesos de riqueza cognitiva, pero además, de naturaleza metacognitiva, afectiva y volitiva. Esta interacción en el aula es un elemento sustancial para caracterizar la CMC, pues es desde este análisis, que se entiende el porqué el estudiante no se puede clasificar a partir de unas pruebas masivas. Al contrario, lo instala en un escenario de instrucción, en el cual se involucra en la interacción entre los sujetos del proceso de enseñanza y aprendizaje, la movilización de la CMC resolviendo problemas significativos de su contexto sociocultural.

La comunicación permitió a los estudiantes comunicar, discutir, argumentar significados matemáticos en la clase. Comprendiendo y compartiendo estos significados mediante ideas conectadas con charlas, exposición de las mismas, de manera escrita como

5 Interacción social en el aula, desarrollo de aspectos comunicacionales verbales y escritos, participación, discusión matemática y negociación compartida de significados matemáticos (Bishop, 2005 y Asfard, 2008). 
de tipo verbal, además, representaron el significado en diversas formas de representación semiótica (símbolos, gráficos, diagramas, algoritmos, etc.); en esta interacción entre profesor-estudiante, estudiante-estudiante, es que el estudiante logra convertirse en miembro de una comunidad matemática, Asfard (2008, p. 29). El estudiante aprendió a comunicarse en el lenguaje de esta comunidad, a compartir sus reglas, a ser parte integral del grupo, a ser un participante activo. La negociación en el desarrollo de los significados matemáticos compartidos asumió la naturaleza de interacción entre los participantes y ayudo al desarrollo de significados matemáticos como un problema de construcción social al interior de la clase de matemáticas Bishop (2005).

\section{Referencias}

Arévalo, A. M. (2012). Comunicación matemática en el aula: uso y gestión de estrategias. Tesis de Maestría, Universidad Católica de la santísima Concepción, Concepción.

Bishop, A.J. (2005). Aproximación sociocultural a la educación matemática, Santiago de Cali, Universidad del Valle.

D’Amore, B; Díaz, J; Fandiño, M. (2008). Competencias y matemáticas. Bogotá, Colombia: cooperativa Editorial magisterio.

Gómez, P. (2002). Análisis didáctico y diseño curricular en matemáticas. Revista EMA, vol. 7, No 3, pp. 251-292.
Jaramillo, D. (2011), La educación matemática en una perspectiva sociocultural: tensiones, utopías, futuros posibles", Revista Educación y Pedagogía, Medellín, Universidad de Antioquia, Facultad de Educación, vol. 23, núm. 59, enero-abril, 2011, pp. 13-36.

OCDE. (2006). PISA. Marco de la evaluación. Conocimientos y habilidades en Ciencias, Matemáticas y Lectura. Santillana Educación S. L. España.

Sfard, A. (2008). Aprendizaje de las matemáticas escolares desde un enfoque comunicacional. Santiago de Cali: Programa editorial Universidad del Valle.

Solar, H. (2009). Competencias de modelización y argumentación en interpretación de gráficas funcionales: propuesta de un modelo de competencias aplicado a un estudio de caso. Tesis doctoral, Universidad Autónoma de Barcelona, Balleterra.

Rico, L; Lupiañez, J.(2008). Competencias matemáticas desde una perspectiva curricular. Madrid, Alianza editorial.

Rojas, F. \& Solar, H. (2011). Instrumentos discursivos para caracterizar la comunicación del profesor en el aula de matemáticas y las posibilidades de participación de los estudiantes. XIII CIAEM-IACME, Recife, Brasil. 